\title{
Parental chromosomal heteromorphisms are not associated with an increased risk of embryo aneuploidy
}

\author{
Carlos Hernandez-Nieto ${ }^{1}$, Sonia Gayete-Lafuente ${ }^{1}$, Tamar Alkon-Meadows ${ }^{1}$, Joseph Lee ${ }^{1}$, Martha Luna-Rojas ${ }^{1}$ \\ Tanmoy Mukherjee ${ }^{1,2}$, Alan B Copperman ${ }^{1,2}$, Benjamin Sandler ${ }^{1,2}$ \\ ${ }^{1}$ Reproductive Medicine Associates of New York, New York, USA \\ 2Obstetrics, Gynecology and Reproductive Science, Icahn School of Medicine at Mount Sinai, New York, USA
}

\begin{abstract}
Objective: Although chromosomal heteromorphisms are commonly found in the general population, some researchers have suggested a correlation with higher rates of embryo aneuploidy. This study aimed to assess the rates of embryo aneuploidy in couples who carry a chromosome heteromorphism.

Methods: The study included couples who had G-banding karyotype testing and underwent an IVF/ PGT-A cycle between January 2012 and March 2018. The participants were classified by couple karyotype: Group $A: \geq 1$ patient reported to be a heterochromatic variant carrier; Group B: both partners reported to be "normal". We assessed the rates of aneuploidy among the groups. We ran a multivariate regression analysis to assess the relationship between heterochromatic variants and the rates of embryo aneuploidy.

Results: Of the 946 couples analyzed, 48 (5.0\%) reported being a carrier of $\geq 1$ heterochromatic variant. We had 869 IVF/PGT-A cycles included in the analysis (Group A: $n=48$; Group B: $n=82$ ). There were no significant differences in embryo ploidy rates among the groups. The heterochromatic chromosome variant was not associated with increased likelihoods of aneuploidy (OR=1.04, CI:95\% $0.85-1.07 ; p=0.46)$. Finally, the gender of the heterochromatic variant carrier had no association with increased likelihood of aneuploidy (OR 1.02, CI 95\% 0.811.28, $p=0.82$ ).

Conclusions: Our study showed no association between parental heterochromatic chromosome variants and subsequent embryo aneuploidy rates. Ploidy rates do not appear to be negatively associated with couples when at least one patient is reported to be a carrier of a heterochromatic variant on the karyotype.
\end{abstract}

Keywords: Preimplantation genetic testing (PGT), embryo aneuploidy, chromosomal heteromorphisms, heterochromatic variants, in vitro fertilization

\section{INTRODUCTION}

Heterochromatic Chromosomal Variants or Chromosomal heteromorphisms $(\mathrm{CH})$ are quantitative or positional alterations in constitutive DNA heterochromatin, occurring in the centromeric region of chromosomes 1, 9, 16, Y, and short arms of acrocentric chromosomes. These alterations or variants, named secondary constriction regions (qh), can include different length patterns for heterochromatin blocks (marked as qh+ or qh-), or even the inversion of an entire block (Mierla \& Stoian, 2012; Šípek et al., 2014). CH were considered to be normal familiar variants due to their frequency in the general population, after karyotyping staining techniques (Dong et al., 2013; Bhasin, 2005; Wyandt \& Tonk, 2012). Today, these heterochromatic regions are known to sustain non-coding repetitive sequences of satellite DNA, which regulates and modulates gene expression under different cellular function conditions. Additionally, these regions contribute to proper chromosome segregation by binding sister centromeres and assembling the kinetochore during meiosis (Skaletsky et al., 2003). Centromere and kinetochore dynamics are essential for correcting or avoiding abnormal chromosome segregation during gamete and/or embryo development processes (Yakin et al., 2005).

The true clinical significance of carrying these heteromorphisms and their impact on human gametes and post-fertilization embryo development has not yet been fully elucidated. Several studies have reported a higher incidence of these variants in couples, affecting both male and females suffering from infertility, recurrent pregnancy loss, or reproductive failure (Šípek et al., 2014; Morales et al., 2016; Düzcan et al., 2003; Madon et al., 2005; Iyer et al., 2007; Sahin et al., 2008; Akbaş et al., 2012; Delhanty et al., 1997; Mantzouratou et al., 2007). However, the relationship between chromosomal heteromorphisms and embryonic chromosomal composition in ART treatments remains predominantly speculative. Thus, the objective of our study is to evaluate whether couples undergoing ART treatments who carry any $\mathrm{CH}$ are at risk of producing embryos with a higher incidence of aneuploidy.

\section{MATERIALS AND METHODS}

\section{Design: Retrospective cohort analysis}

The study included couples with G-banding karyotype testing on peripheral blood lymphocytes who underwent an IVF/ICSI cycle, with preimplantation genetic testing for aneuploidy (PGT-A), from 2012 to 2018. The couples were separated into groups based on their karyotype results (Group A: $\geq 1$ patient reported to be a heterochromatic variant carrier; Group B: both partners reported to be "cytogenetically normal"). Indications for karyotype testing and PGT-A included: patients with a history of pregnancy losses, aneuploidy, prior stillbirth, poor embryonic quality, and severe male factor infertility. Couples in which one or both partners were found to be carriers of any balanced translocation, chromosomal inversions, mosaicisms, or known pathogenic polymorphisms were excluded from the analysis. Additionally, patients diagnosed with severe male factor and those who required testicular sperm extraction and/or ovum donation were excluded from the study.

\section{IVF and laboratory procedures}

The patients underwent ovarian stimulation for IVF, all stimulation protocols, laboratory procedures, and embryo morphology grading specifics have been previously described elsewhere (Hernandez-Nieto et al., 2019). All oocytes were inseminated with ICSI and underwent extended culture to the blastocyst stage of development. Trophectoderm (TE) biopsies were submitted to chromosome copy number analysis through quantitative real-time PCR (qPCR), and/or next generation sequencing (NGS) based 
analysis. The biopsy results were interpreted as euploid, aneuploid, or inconclusive result, as described previously (Hernandez-Nieto et al., 2017), mosaic embryos were catalogued as aneuploid. All inconclusive result embryos that underwent a second TE biopsy were excluded from the analysis.

Euploidy rate was defined as the number of euploid embryos over the number of embryos biopsied.

\section{Statistical methods}

The statistical analysis was performed using SAS version 9.4 (SAS institute Inc., Cary, NC, USA). The descriptive data was compared by T-test, $\chi^{i^{2}}$ test and Mann-Whitney $U$ tests when appropriate. All results were expressed as percentages, means and Standard Deviations (SD). Adjusted odds ratios (OR) with $95 \%$ CI were calculated using a multivariate logistic regression analysis to assess the effect of a $\mathrm{CH}$ on any of the patients and the odds of increased embryo aneuploidy.

The logistic regression models were fitted with generalized estimating equations (GEE) to account for patients who underwent multiple cycles. All variables that showed significance and were thought to be clinically relevant were included as covariates in the model. All p-values are two-sided with a clinical significance level of $p<0.05$.

\section{RESULTS}

869 couples underwent karyotype testing, of those, 48 $(5.0 \%)$ were found to be a carrier of a $\mathrm{CH}$. There were no couples in which both partners carried a $\mathrm{CH}$ in the study. The most common $\mathrm{CH}$ types found were non-pathogenic pericentric inversions on chromosome $9(15 / 48,31.2 \%)$ and block variants ( $q$ h) on chromosome $\mathrm{Y}(11 / 48,22.9 \%)$. $\mathrm{CH}$ were more common in male partners $(32 / 48,66.6 \%)$ than in females $(16 / 48 ; 33.3 \%)$. All the cases had heterozygosity in the $\mathrm{CH}$ carrier. A total of 869 IVF/PGT-A cycles were included in the analysis (Group A "carriers": $n=48$; Group B "normal karyotypes": $n=821$ ). Of those cases, 4,017 trophectoderm biopsies were analyzed (Group A: $n=264$; Group B: 3753). Demographic, stimulation parameters and embryological variables were comparable among cohorts (Table 1).
Oocyte maturity rates between $\mathrm{CH}$ carriers and non-carriers were comparable (Group A: $77.40 \%$; Group: $80.30 \% ; p=0.91$ ); similarly, fertilization rates (Group A: $77.80 \%$; Group B: $80.30 \%$; $p=0.61)$; blastulation rates (Group A: 71.20\%; Group B: $64.60 \% ; p=0.19$ ) and mean number of biopsied/cryopreserved blastocysts per cycle (Group A: $82.50 \%$; Group B: $74.10 \%, p=0.21$ ). In a multivariate regression analysis adjusted for female and male patients' age, BMI, and AMH levels, there was no association with the presence of a $\mathrm{CH}$ variant and lower odds of blastulation (OR 1.85, CI 95\% 0.56-6.10). There were no significant differences in embryo euploidy rates (Group A: $45 \%$; Group B: $52 \% ; p=0.61$ ), aneuploidy rates (A: $48 \%$; B: $44 \% ; p=0.98)$ or inconclusive report rates (A: 7\%; $\mathrm{B}$ : $3.7 \% ; p=0.32$ ) (Table 2).

In a multivariate regression analysis adjusted for female and male patients age, body mass index, $\mathrm{AMH}$, and day when embryo biopsy was performed. There was no association with the presence of a $\mathrm{CH}$ variant and increased odds of aneuploidy (OR=1.04, CI95\% $0.92-1.18)$. A sub-analysis adjusted for gender and $\mathrm{CH}$ carrier status found no association with the presence of a $\mathrm{CH}$ and increased odds of embryo aneuploidy when comparing male versus female carriers (OR 1.02, CI 95\% 0.81-1.28).

\section{DISCUSSION}

As heterochromatin plays an essential role in meiosis, the presence of $\mathrm{CH}$ has been theorized to impair the formation of functional gametes. Consequently, patients who are $\mathrm{CH}$ carriers might theoretically be more susceptible to experiencing an increased incidence of embryonic aneuploidy and impaired reproductive outcome (Morales et al., 2016). Our study suggests that couples carrying $\mathrm{CH}$ are not at greater risk of experiencing increased rates of embryo aneuploidy when compared to non-carriers. The most common $\mathrm{CH}$ found in this study involved pericentric inversions of chromosome $9(31.2 \%)$; in second, variants in detectable regions of the $Y$ chromosome $(22.9 \%)$. According to the literature, chromosome 9 pericentric inversions are the most frequent $\mathrm{CH}$ in infertile patients, but also in the general population, followed by $9 q h+$ and 9qh- variants

Table 1. Comparison analysis of demographic, oocyte stimulation, embryological and ploidy variables of the populations analyzed.

\begin{tabular}{|c|c|c|c|}
\hline Variable & $\begin{array}{l}\text { Variant carrier } \\
n=48 \text { cycles }\end{array}$ & $\begin{array}{c}\text { Normal Karyotype } \\
n=821 \text { cycles }\end{array}$ & $p$-value \\
\hline Age (years) & $36.22 \pm 4.22$ & $36.37 \pm 4.16$ & 0.81 \\
\hline Body Mass Index $\left(\mathrm{Kg} / \mathrm{m}^{2}\right)$ & $22.82 \pm 3.61$ & $28.42 \pm 143.12$ & 0.27 \\
\hline Day of ovulation trigger & $12.06 \pm 1.42$ & $12.91 \pm 13.88$ & 0.11 \\
\hline Gonadotropin Cumulative Dose (IU) & $3988.47 \pm 1562.28$ & $3769.04 \pm 1337.67$ & 0.27 \\
\hline Estradiol at trigger $(\mathrm{pg} / \mathrm{mL})$ & $2321.91 \pm 880.83$ & $2447.39 \pm 1170.89$ & 0.35 \\
\hline Progesterone at trigger $(\mathrm{ng} / \mathrm{mL})$ & $0.86 \pm 0.42$ & $0.94 \pm 0.54$ & 0.27 \\
\hline Baseline FSH (IU/mL) & $6.44 \pm 3.78$ & $6.09 \pm 3.37$ & 0.57 \\
\hline Anti-Mullerian Hormone (ng/mL) & $3.70 \pm 4.65$ & $3.28 \pm 3.45$ & 0.52 \\
\hline Antral follicle count $(n)$ & $12.23 \pm 6.68$ & $12.33 \pm 5.97$ & 0.91 \\
\hline Oocytes Retrieved (n) & $15.85 \pm 9.56$ & $15.77 \pm 8.49$ & 0.95 \\
\hline Mature (MII) oocytes (n) & $12.02 \pm 8.11$ & $11.78 \pm 7.07$ & 0.81 \\
\hline 2PN embryos $(n)$ & $9.35 \pm 6.89$ & $9.54 \pm 6.03$ & 0.83 \\
\hline Blastocysts biopsied ( $\mathrm{n}$ ) & $5.62 \pm 4.67$ & $4.69 \pm 3.67$ & 0.18 \\
\hline Aneuploid embryos (n) & $2.70 \pm 2.60$ & $2.08 \pm 1.89$ & 0.11 \\
\hline Euploid embryos ( $n$ ) & $2.57 \pm 2.73$ & $2.44 \pm 2.67$ & 0.73 \\
\hline Inconclusive results $(\mathrm{n})$ & $0.33 \pm 0.93$ & $0.17 \pm 0.63$ & 0.24 \\
\hline
\end{tabular}

Note: Data presented as means \pm standard deviation, unless stated otherwise. Statistical significance, $p<0.05$. 
Table 2. Outcomes of oocytes retrieved and ploidy rates of the populations analyzed.

\begin{tabular}{|c|c|c|c|c|c|}
\hline \multirow{2}{*}{ Variable outcome } & \multicolumn{2}{|c|}{ Variant Carrier } & \multicolumn{2}{|c|}{ Normal Karyotype } & \multirow{2}{*}{$p$-value } \\
\hline & $\mathbf{N}$ & $\%$ & $\mathbf{N}$ & $\%$ & \\
\hline Oocytes retrieved & 745 & & 12633 & & 0.95 \\
\hline Mature oocytes rate & $577 / 745$ & $77.40 \%$ & $9750 / 12633$ & $77.10 \%$ & 0.95 \\
\hline Fertilization rate & $449 / 577$ & $77.80 \%$ & $7835 / 9750$ & $80.30 \%$ & 0.61 \\
\hline Blastulation rate & $320 / 449$ & $71.20 \%$ & $5064 / 7835$ & $64.60 \%$ & 0.19 \\
\hline Biopsied blastocyst rate & $264 / 320$ & $82.50 \%$ & $3753 / 5064$ & $74.10 \%$ & 0.21 \\
\hline Euploid embryos rate & $121 / 264$ & $45 \%$ & $1951 / 3753$ & $52 \%$ & 0.61 \\
\hline Aneuploid embryos rate & $127 / 264$ & $48 \%$ & $1660 / 3753$ & $44 \%$ & 0.98 \\
\hline Inconclusive reports rate & $20 / 264$ & $7 \%$ & $142 / 3753$ & $3.70 \%$ & 0.32 \\
\hline
\end{tabular}

Note: Data presented as percentages, unless stated otherwise. Statistical significance, $p<0.05$.

(Ferguson-Smith, 1974; Verma et al., 1978; Humphray et al., 2004; Codina-Pascual et al., 2006; Collodel et al., 2006; Penna Videaú et al., 2001; Nagvenkar et al., 2005; Ferguson et al., 2007; 2009; Sarrate et al., 2014; GarcíaPeiró et al., 2011a; 2011b).

Although some studies have addressed the potential associations of $\mathrm{CH}$ on fertility treatments, our study found no differences in ovarian stimulation parameters, number of oocytes retrieved and fertilization rates during IVF/ICSI cycles. Conversely, one study by Guo et al. (2012) found lower fertilization rates in $\mathrm{CH}$-carrying men with severe oligozoospermia, compared with non-carriers also with severe oligozoospermia. Thus, suggesting that $\mathrm{CH}$ might have detrimental effects on spermatogenesis and a negative impact on IVF outcomes (Guo et al., 2012). Additionally, Liang et al. (2014) reported lower fertilization rates in couples in which only the male carried the $\mathrm{CH}$, compared with couples with only a female $\mathrm{CH}$-carrier and infertile couples with normal karyotypes. Notably, these aforementioned studies included cohorts of mixed conventional IVF and ICSI cases. In our study, all cases underwent ICSI as insemination method, and our results showed similar fertilization rates among patients who were $\mathrm{CH}$ carriers and non-carriers ( $80.3 \%$ vs. $77.8 \%, p=0.61)$.

Our data suggests that embryonic blastulation, embryonic morphological quality and euploidy rates are not significantly associated with the presence or absences of $\mathrm{CH}$ on couple's karyotypes. A study by Xu et al. (2016) reported that $\mathrm{CH}$ in either male or female carriers seemed to have adverse effects on IVF/ICSI-ET outcomes. That study suggested that $\mathrm{CH}$ in male carriers affected IVF outcomes by decreasing the rates of fertilization, number of available cleavage stage embryos, good quality embryos and clinical outcomes after the transfer of these embryos. In addition, the presence of $\mathrm{CH}$ in female carriers affected outcomes only by lowering the embryo cleavage rate. To our knowledge, this deleterious effect has yet to be reported for extended culture of the embryo to the blastocyst stage. Our study is the first to demonstrate that blastulation rates $(64.4 \%$ vs. $71.2 \%, p=0.19)$ and total number of cryopreserved and biopsied blastocysts $(74.1 \%$ vs. $82.5 \%$, $p=0.21$ ) were not adversely affected by $\mathrm{CH}$ carrier status, regardless of paternal or maternal contribution.

A retrospective analysis by Morales et al. (2016), examined the relationship between $\mathrm{CH}$, infertility and aneuploidy in sperm cells and embryos. Apart from observing a high prevalence of $\mathrm{CH}$ among infertile patients when compared to a control group, $(19.4 \%$ vs. $13.4 \%$; $p<0.01)$, they reported major rates of sperm aneuploidy in $\mathrm{CH}$ carriers $(37.7 \%$ vs. $16.3 \% ; p<0.01)$. Further, they found an increased rate of embryo aneuploidy rates in female carriers than in non-carrier oocyte recipients (102 embryos from $\mathrm{CH}$ carriers with a reported aneuploidy rate of $50.0 \%$ compared with 199 embryos of a control group yielding a $27.6 \%$ aneuploidy rate $(p<0.001)$. Conversely, after analyzing 4,017 blastocysts, our study's results showed no significant differences in embryo ploidy rates among groups. Furthermore, after utilizing an adjusted analysis controlling for patient's age and other potential confounders, there was no association with the presence of a $\mathrm{CH}$ variant and increased odds of embryo aneuploidy. Additionally, there was no association with the odds of aneuploidy after performing a sub-analysis that adjusted for the sex of the $\mathrm{CH}$ carrier. One of the main differences from our study compared to that from Morales et al. (2016), was our use of the most contemporary technology available for genetic assessment. While they examined the embryos by a-CGH, we analyzed embryonic chromosomal composition with more modern clinically validated platforms including NGS (Friedenthal et al., 2018; Lee et al., 2015).

Despite our best effort to avoid biases on the study, this analysis still has limitations, as its retrospective nature increases the likelihood of a selection bias. One limitation to consider is the lack of standardization for karyotyping indications in patients and couples who undergo ART. The cytogenetically karyotyped individuals in our study included patients with refractory infertility or prior history of poor IVF outcomes or embryonic quality, or couples with a history of recurrent losses and implantation failure. Also, not all the karyotyping was performed in a same laboratory. Some commercial laboratories do not consider $\mathrm{CH}$ as normal variants and exclude from their report, differences in reporting might underestimate the $\mathrm{CH}$ prevalence in the general population. Finally, we did not include the pregnancy or perinatal outcomes of the studied embryos in our study, as these outcomes were beyond the scope of our analysis.

One of the strengths of our study is that all the clinical data analyzed came from a single, high volume center experienced in blastocyst trophectoderm biopsy. Our study includes one of the largest cohort of embryos chromosomally screened in couples carrying a $\mathrm{CH}$, also, we compared the embryo aneuploidy rate from all infertile patients with multiple infertility diagnoses ( $\mathrm{CH}$ carrier vs non-carrier). Moreover, we excluded clinical diagnoses associated with a significant increase in aneuploid embryos during ART such as carriers of balanced translocations, inversions, mosaicisms, or known pathogenic polymorphisms (Petracchi et al., 2009; Warburton \& Fraser, 1964; Hassold et al., 1980). Concordant to previous published studies, we excluded patients with severe male factor utilizing testicular sperm extraction, these group of patients had been previously 
reported to be associated with a higher frequency of $Y$ chromosome variants compared to fertile men (Morales et al., 2016; Penna Videaú et al., 2001; Nagvenkar et al., 2005; Guo et al., 2012) and with an increased rate of sperm aneuploidy (Yakin et al., 2005; Morales et al., 2016; Ferguson et al., 2007; García-Peiró et al., 2011a; 2011b; Mozdarani et al., 2007; Minocherhomji et al., 2009); both contributors to an increased risk of embryonic aneuploidy. Finally, our study utilized an adjusted multivariate analysis fitted with a GEE that accounted for patients who underwent multiple cycles and controlled for other potential cofounders (i.e. patients age, BMI, AMH, ovarian stimulation parameters, and embryological variables).

The relatively high incidence of $\mathrm{CH}$ observed in infertile patients demands the need to evaluate the potential associations with infertility and subfertility. The relationship between 'normal' $\mathrm{CH}$ and reproductive outcome remains highly contested; epigenetic, genetic, and chromosomal modifications have been associated with infertility and poor reproductive outcomes (Morales et al., 2016; Collodel et al., 2006; Penna Videaú et al., 2001; Nagvenkar et al., 2005; Ferguson et al., 2007; 2009; Sarrate et al., 2014; García-Peiró et al., 2011a; 2011b). However, the findings of our study showed that there is no association between parental $\mathrm{CH}$ and increased risk of embryonic aneuploidy. Ploidy rates do not appear to be negatively associated with couples when at least one patient is reported to be a carrier of a heterochromatic variant on karyotype. Further analysis with high-resolution genome karyomapping and haplotyping technology may unveil potential relationships between parental chromosomal variants and embryological chromosome segregation errors and give us further insight on their interaction with embryonic development.

\section{ACKNOWLEDGEMENTS}

The authors thank all the physicians, fellows, embryologists, research and staff members for the valuable work and help in making this manuscript.

\section{Ethics approval}

The Western Institutional Review approved this retrospective analysis (WIRB PRO NUM: 20161791; Study Number: 1167398). The study complies with all ethical standards for medical research, all patient information was made anonymous, and the patients had their identities secured prior to the analysis.

\section{Declaration of interest statement}

We received no funding to carry out this study. Dr. A.B.C. is advisor and/or board member of Sema4 (Stakeholder in data), Progyny and Celmatix. The other authors have no conflicts of interest to declare.

\section{CONFLICT OF INTEREST}

The authors have no conflict of interest to declare.

\section{Corresponding author:}

Carlos A Hernandez-Nieto

Reproductive Medicine Associates of New York, LLP

New York, New York 10022, USA

E-mail: chernandez@rmany.com

ORCID: https://orcid.org/0000-0002-6703-1341

\section{REFERENCES}

Akbaş H, Isi H, Oral D, Türkyılmaz A, Kalkanlı-Taş S, Simşek S, Balkan M, Sakar MN, Fidanboy M, Alp MN, Budak T. Chromosome heteromorphisms are more frequent in couples with recurrent abortions. Genet Mol Res. 2012;11:384751. PMID: 23212323 DOI: 10.4238/2012. November.12.1

Bhasin MK. Human population cytogenetics: a review. Int J Hum Genet. 2005;5:83-152. DOI: $10.1080 / 09723757.2005 .11885918$

Codina-Pascual M, Navarro J, Oliver-Bonet M, Kraus J, Speicher MR, Arango O, Egozcue J, Benet J. Behaviour of human heterochromatic regions during the synapsis of homologous chromosomes. Hum Reprod. 2006;21:1490-7. PMID: 16484310 DOI: 10.1093/humrep/del028

Collodel G, Moretti E, Capitani S, Piomboni P, Anichini C, Estenoz M, Baccetti B. TEM, FISH and molecular studies in infertile men with pericentric inversion of chromosome 9. Andrologia. 2006;38:122-7. PMID: 16872463 DOI: 10.1111/j.1439-0272.2006.00725.x

Delhanty JD, Harper JC, Ao A, Handyside AH, Winston RM. Multicolour FISH detects frequent chromosomal mosaicism and chaotic division in normal preimplantation embryos from fertile patients. Hum Genet. 1997;99:755-60. PMID: 9187668 DOI: $10.1007 / \mathrm{s} 004390050443$

Dong Y, Jiang YT, Du RC, Zhang HG, Li LL, Liu RZ. Impact of chromosomal heteromorphisms on reproductive failure and analysis of 38 heteromorphic pedigrees in Northeast China. J Assist Reprod Genet. 2013;30:275-81. PMID: 23274509 DOI: $10.1007 / \mathrm{s} 10815-012-9910-z$

Düzcan F, Atmaca M, Cetin GO, Bagci H. Cytogenetic studies in patients with reproductive failure. Acta Obstet Gynecol Scand. 2003;82:53-6. PMID: 12580840 DOI: 10.1034/j.1600-0412.2003.820109.x

Ferguson KA, Wong EC, Chow V, Nigro M, Ma S. Abnormal meiotic recombination in infertile men and its association with sperm aneuploidy. Hum Mol Genet. 2007;16:2870-9. PMID: 17728321 DOI: $10.1093 / \mathrm{hmg} / \mathrm{ddm} 246$

Ferguson KA, Leung S, Jiang D, Ma S. Distribution of MLH1 foci and inter-focal distances in spermatocytes of infertile men. Hum Reprod. 2009;24:1313-21. PMID: 19246465 DOI: $10.1093 /$ humrep/dep021

Ferguson-Smith MA. Autosomal polymorphisms. Birth Defects Orig Artic Ser. 1974;10:19-29. PMID: 4142589

Friedenthal J, Maxwell SM, Munné S, Kramer $\mathrm{Y}$, McCulloh $\mathrm{DH}$, McCaffrey C, Grifo JA. Next generation sequencing for preimplantation genetic screening improves pregnancy outcomes compared with array comparative genomic hybridization in single thawed euploid embryo transfer cycles. Fertil Steril. 2018;109:627-32. PMID: 29605407 DOI: $10.1016 /$ j.fertnstert.2017.12.017 
García-Peiró A, Oliver-Bonet M, Navarro J, Abad C, Guitart M, Amengual MJ, Gosálvez J, Benet J. Dynamics of sperm DNA fragmentation in patients carrying structurally rearranged chromosomes. Int J Androl. 2011a;34:e546-53. PMID: 21535010 DOI: 10.1111/j.1365-2605.2011.01153.x

García-Peiró A, Oliver-Bonet M, Navarro J, Abad C, Guitart $M$, Amengual MJ, Benet J. Sperm DNA integrity and meiotic behavior assessment in an infertile male carrier of a $9 \mathrm{qh}+++$ polymorphism. J Biomed Biotechnol. 2011b;2011:730847. PMID: 21197455 DOI: 10.1155/2011/730847

Guo T, Qin Y, Gao X, Chen H, Li G, Ma J, Chen ZJ. The role of male chromosomal polymorphism played in spermatogenesis and the outcome of IVF/ICSI-ET treatment. Int J Androl. 2012;35:802-9. PMID: 22712895 DOI: 10.1111/j.1365-2605.2012.01284.x

Hassold T, Chen N, Funkhouser J, Jooss T, Manuel B, Matsuura J, Matsuyama A, Wilson C, Yamane JA, Jacobs PA. A cytogenetic study of 1000 spontaneous abortions. Ann Hum Genet. 1980;44:151-78. PMID: 7316468 DOI: 10.1111/j.1469-1809.1980.tb00955.x

Hernandez-Nieto C, Lee J, Nazem T, Gounko D, Copperman A, Sandler B. Embryo aneuploidy is not impacted by selective serotonin reuptake inhibitor exposure. Fertil Steril. 2017;108:9739. PMID: 29202974 DOI: 10.1016/j.fertnstert.2017.08.040

Hernandez-Nieto C, Lee JA, Slifkin R, Sandler B, Copperman $A B$, Flisser $E$. What is the reproductive potential of day 7 euploid embryos? Hum Reprod. 2019;34:1697-706. PMID: 31398251 DOI: 10.1093/humrep/dez129

Humphray SJ, Oliver K, Hunt AR, Plumb RW, Loveland JE, Howe KL, Andrews TD, Searle S, Hunt SE, Scott CE, Jones $M C$, Ainscough R, Almeida JP, Ambrose KD, Ashwell RI, Babbage AK, Babbage S, Bagguley CL, Bailey J, Banerjee $\mathrm{R}$, et al. DNA sequence and analysis of human chromosome 9. Nature. 2004;429:369-74. PMID: 15164053 DOI: $10.1038 /$ nature 02465

Iyer $\mathrm{P}$, Wani L, Joshi S, Lakshmi J, Dalvi R, Chavan D, Das $B R$, Mandava S. Cytogenetic investigations in couples with repeated miscarriages and malformed children: report of a novel insertion. Reprod Biomed Online. 2007;14:314-21. PMID: 17359584 DOI: $10.1016 / S 1472-6483(10) 60873-5$

Lee $E$, Illingworth P, Wilton L, Chambers GM. The clinical effectiveness of preimplantation genetic diagnosis for aneuploidy in all 24 chromosomes (PGD-A): systematic review. Hum Reprod. 2015;30:473-83. PMID: 25432917 10.1093/humrep/deu303

Liang J, Zhang Y, Yu Y, Sun W, Jing J, Liu R. Effect of chromosomal polymorphisms of different genders on fertilization rate of fresh IVF-ICSI embryo transfer cycles. Reprod Biomed Online. 2014;29:436-44. PMID: 25131557 DOI: 10.1016/j.rbmo.2014.06.011

Madon PF, Athalye AS, Parikh FR. Polymorphic variants on chromosomes probably play a significant role in infertility. Reprod Biomed Online. 2005;11:726-32. PMID: 16417737 DOI: $10.1016 / \mathrm{S} 1472-6483(10) 61691-4$
Mantzouratou A, Mania A, Fragouli E, Xanthopoulou L, Tashkandi S, Fordham K, Ranieri DM, Doshi A, Nuttall S, Harper JC, Serhal P, Delhanty JD. Variable aneuploidy mechanisms in embryos from couples with poor reproductive histories undergoing preimplantation genetic screening. Hum Reprod. 2007;22:1844-53. PMID: 17502322 DOI: 10.1093/ humrep/dem 102

Mierla D, Stoian V. Chromosomal polymorphisms involved in reproductive failure in the romanian population. Balkan J Med Genet. 2012;15:23-8. PMID: 24052728 DOI: 10.2478/bjmg-2013-0003

Minocherhomji S, Athalye AS, Madon PF, Kulkarni D, Uttamchandani SA, Parikh FR. A case-control study identifying chromosomal polymorphic variations as forms of epigenetic alterations associated with the infertility phenotype. Fertil Steril. 2009;92:88-95. PMID: 18692838 DOI: $10.1016 /$ j.fertnstert.2008.05.071

Morales R, Lledó B, Ortiz JA, Ten J, Llácer J, Bernabeu R. Chromosomal polymorphic variants increase aneuploidies in male gametes and embryos. Syst Biol Reprod Med. 2016;62:317-24. PMID: 27560353 DOI: 10.1080/19396368.2016.1212949

Mozdarani $\mathrm{H}$, Meybodi AM, Karimi $\mathrm{H}$. Impact of pericentric inversion of Chromosome 9 [inv (9) (p11q12)] on infertility. Indian J Hum Genet. 2007;13:26-9. PMID: 21957338 DOI: $10.4103 / 0971-6866.32031$

Nagvenkar P, Desai K, Hinduja I, Zaveri K. Chromosomal studies in infertile men with oligozoospermia \& non-obstructive azoospermia. Indian J Med Res. 2005;122:34-42. PMID: 16106088

Penna Videaú S, Araujo H, Ballesta F, Ballescá JL, Vanrell JA. Chromosomal abnormalities and polymorphisms in infertile men. Arch Androl. 2001;46:205-10. PMID: 11339646 DOI: $10.1080 / 01485010151096504$

Petracchi F, Colaci DS, Igarzabal L, Gadow E. Cytogenetic analysis of first trimester pregnancy loss. Int J Gynaecol Obstet. 2009;104:243-4. PMID: 19036372 DOI: 10.1016/j.ijgo.2008.10.014

Sahin FI, Yilmaz Z, Yuregir OO, Bulakbasi T, Ozer O, Zeyneloglu HB. Chromosome heteromorphisms: an impact on infertility. J Assist Reprod Genet. 2008;25:191-5. PMID: 18461436 DOI: $10.1007 /$ s10815-008-9216-3

Sarrate Z, Vidal F, Blanco J. Meiotic abnormalities in metaphase I human spermatocytes from infertile males: frequencies, chromosomes involved, and the relationships with polymorphic karyotype and seminal parameters. Asian J Androl. 2014;16:838-44. PMID: 25080930 DOI: $10.4103 / 1008-682 X .135126$

Šípek A Jr, Mihalová R, Panczak A, Hrčková L, Janashia $M$, Kaspríková N, Kohoutová M. Heterochromatin variants in human karyotypes: a possible association with reproductive failure. Reprod Biomed Online. 2014;29:245-50. PMID: 24928354 DOI: 10.1016/j. rbmo.2014.04.021 
Skaletsky $\mathrm{H}$, Kuroda-Kawaguchi $\mathrm{T}$, Minx PJ, Cordum HS, Hillier L, Brown LG, Repping S, Pyntikova T, Ali J, Bieri T, Chinwalla A, Delehaunty A, Delehaunty K, Du H, Fewell G, Fulton L, Fulton R, Graves T, Hou SF, Latrielle $P$, et al. The male-specific region of the human $Y$ chromosome is a mosaic of discrete sequence classes. Nature. 2003;423:82537. PMID: 12815422 DOI: $10.1038 /$ nature01722

Verma RS, Dosik H, Lubs HA. Size and pericentric inversion heteromorphisms of secondary constriction regions $(h)$ of chromosomes 1, 9, and 16 as detected by CBG technique in Caucasians: classification, frequencies, and incidence. Am J Med Genet. 1978;2:331-9. PMID: 263447 DOI: 10.1002/ajmg.1320020403

Warburton D, Fraser FC. Spontaneous abortion risks in man: Data from reproductive histories collected in a medical genetics unit. Am J Hum Genet. 1964;16:1-25. PMID: 14131871
Wyandt HE, Tonk VS. Human chromosome variation: heteromorphism and polymorphism. Dordrecht: Springer Netherlands; 2012.

Xu X, Zhang R, Wang W, Liu H, Liu L, Mao B, Zeng X, Zhang $X$. The effect of chromosomal polymorphisms on the outcomes of fresh IVF/ICSI-ET cycles in a Chinese population. J Assist Reprod Genet. 2016;33:1481-6. PMID: 27544276 DOI: $10.1007 / \mathrm{s} 10815-016-0793-2$

Yakin K, Balaban B, Urman B. Is there a possible correlation between chromosomal variants and spermatogenesis? Int J Urol. 2005;12:984-9. PMID: 16351655 DOI: 10.1111/j.1442-2042.2005.01185.x 